\title{
Monolithic 2-D Scanning Mirror Using Self-Aligned Angular Vertical Comb Drives
}

\author{
Jongbaeg Kim, Dane Christensen, and Liwei Lin, Member, IEEE
}

\begin{abstract}
We have demonstrated microfabricated, monolithic two degrees of freedom (two-dimensional) electrostatic torsional mirrors using a three-mask process on silicon-on-insulator wafer with a single plastic deformation step. The mirror operated independently in two orthogonal directions as controlled by two sets of self-aligned angular vertical combs. The measured dynamic performance showed resonant frequencies of 10.56 and $1.54 \mathrm{kHz}$ with optical scanning angles up to $27^{\circ}$ and $20^{\circ}$ in the two orthogonal axes, respectively, under driving voltages of $20 \mathrm{~V}_{\mathrm{dc}}$ plus $15 \mathrm{~V}_{\mathrm{pp}}$. A 90-day continuous mirror operation at peak resonance, in equivalent to 80 and 12.1 billion cycles on the two orthogonal axes, showed negligible performance variations.
\end{abstract}

Index Terms-Angular vertical comb, microelectromechanical systems, plastic deformation, scanning micromirror, two-dimensional (2-D) microactuator.

\section{INTRODUCTION}

A PPLICATIONS in optical microelectromechanical systems have utilized vertically aligned comb-drives to excite torsional actuations [1]-[4]. Particularly, mirrors with two degrees of freedom are sought for various applications such as area scanning in display/sensing systems and channel switching in telecommunication devices. Previous approaches include using two uniaxial mirrors that are orthogonally aligned, or one monolithic two-dimensional (2-D) mirror independently controlled in each orthogonal direction. The latter is more advantageous as it eliminates additional assembly and alignment step, requires smaller physical space, and minimizes the light power dissipation without going through the reflection process necessary for the two-mirror approach.

Previous work for monolithic 2-D actuators include means of thermal [5], electromagnetic [6], and electrostatic [7], [8] actuation. Thermal actuators are typically limited to low operation frequency and larger power consumption [5]. Electromagnetic actuators [6] resulted in bulky structures with the assembly of permanent magnet and/or complicated fabrication processes. Several electrostatically driven 2-D micromirrors were reported previously. Some are based on parallel plate actuators [7] with the drawbacks of pull-in phenomenon. Others utilized electrostatic levitation forces [8] with a limited dc operation range. Piyawattanametha et al. [9] demonstrated vertical comb based 2-D actuator using manual assembly of the microstructures and combination of both surface and bulk micromachining. Kwon et $a l$. [10] reported vertical comb-drives with rather complicated

Manuscript received July 7, 2005.

J. Kim is with the School of Mechanical Engineering, Yonsei University, Seoul 120-749, Korea (e-mail: kimjb@yonsei.ac.kr).

D. Christensen and L. Lin are with the Berkeley Sensor and Actuator Center, University of California, Berkeley, CA 94720 USA.

Digital Object Identifier 10.1109/LPT.2005.857612

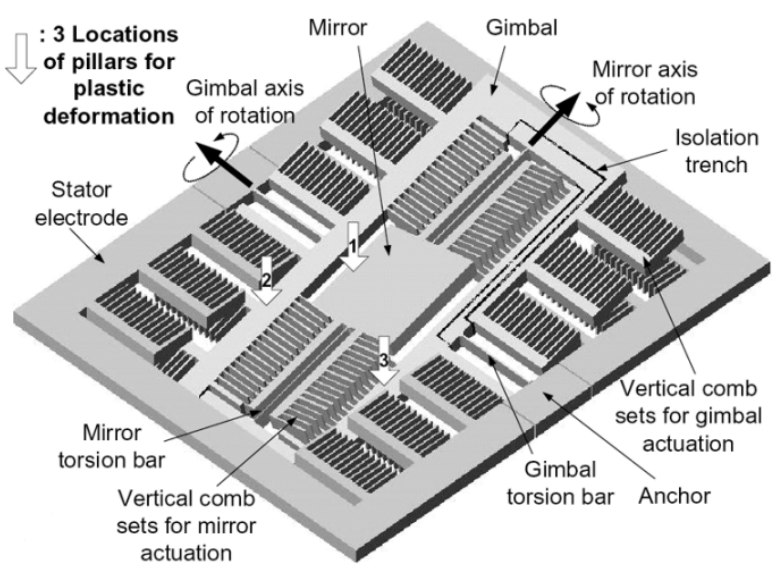

Fig. 1. Schematic view of the plastically deformed 2-D scanning mirror actuator with self-aligned vertically interdigitated comb sets.

fabrication process where wafer bonding and multiple times of deep reactive ion etching (DRIE) of silicon-on-insulator (SOI) are necessary. Torsional actuators with linkage-based design have also been demonstrated [11], [12], where mirrors are connected to a number of linkage structures through which actuation forces are transferred. The additional compliances introduced by linkages typically make the linkage-based 2-D mirrors less resistant to shock and vibration, which could affect the optical performance of the mirrors.

In this letter, we present batch-processed single crystalline silicon monolithic 2-D scanning micromirrors with linkage-less design using gimbal structure. The self-aligned plastic deformation process developed for one-dimensional scanning micromirrors [3], [4] is adopted and extended for the 2-D actuator as illustrated in Fig. 1. For independently controlled 2-D motion, the mirror and gimbal structure should be electrically isolated and mechanically connected, and we used trench refill and etch back [8], [13] for this.

\section{DESIGN AND FABRICATION}

Three pillars, as shown in Fig. 1, at the designated location will strain both gimbal and mirror torsion bars simultaneously in a self-aligned plastic deformation process to provide permanently tilted angles with respect to the gimbal- and mirror axis [3]. Pillar 1 pushes down the mirror edge such that the mirror rotates in the direction of "mirror axis of rotation," forming the tilt angle between mirror and gimbal frame. At the same time, Pillars 2 and 3 push down the gimbal such that the whole gimbal rotates with respect to the "gimbal axis of rotation" to form a tilt angle. Plastic deformation is achieved by straining both gimbal and mirror torsion bars simultaneously as described and heating them to a high temperature to relieve induced stresses and leave 

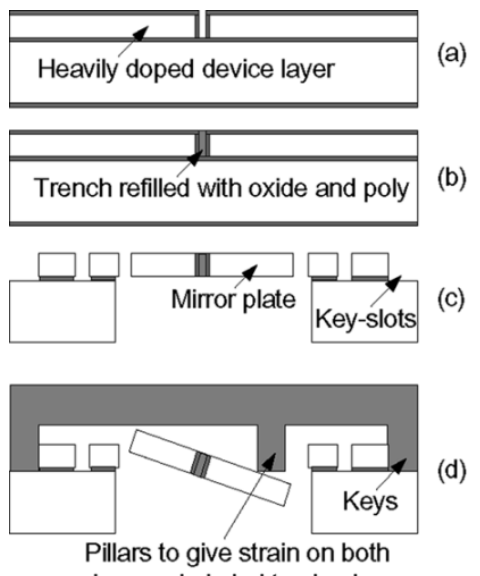
mirror and gimbal torsion bars

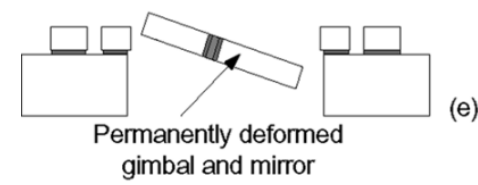

Fig. 2. Fabrication process flow for plastically deformed 2-D scanning mirror actuators. (a) Trench etch and oxidation (for electrical isolation). (b) Poly deposition (for mechanical connection) etch-back. (c) Pattern and DRIE on both sides followed by etch oxide to release the structure. d) Place separately prepared lid wafer on op of device wafer and anneal at high emperature $\left(900^{\circ} \mathrm{C}\right)$. (e) Separate the device wafer and the lid wafer (flexures are deformed permanently).

permanent deformation on both pairs of torsion bars. The detailed mechanism of the plastic deformation of silicon structures are found in the [3], [4]. Therefore, the mirror is tilted with respect to two orthogonal axes allowing two sets of vertically interdigitated comb fingers to be positioned at different heights to control rotational motions in the orthogonal axes independently. The fabricated device is composed of $650 \times 650 \times 50 \mu \mathrm{m}^{3}$ size mirror and $700 \times 10 \times 50 \mu \mathrm{m}^{3}$ and $500 \times 10 \times 50 \mu \mathrm{m}^{3}$ size torsional springs on the mirror and gimbal axes, respectively. The total number of comb pairs is 128 for the mirror and 288 for the gimbal actuation, the overlapping length of opposite comb fingers is $200 \mu \mathrm{m}$, and the gap between stationary comb and movable comb is $8 \mu \mathrm{m}$. The radius of curvature of the single crystalline mirror measured using white light interferometer is in the order of meter. The moving parts, gimbal and mirror, are symmetric and comb fingers are on both sides of torsion bar as shown. Symmetric rotor configuration with balanced moment of mass is advantageous for the robustness of the device.

The actuators require three electrodes to control actuation in the two orthogonal directions independently. The gimbal and the comb structures attached to it are grounded, and the mirror and the stator are biased differently. The movable electrodes, gimbal, and mirror should be mechanically connected but electrically isolated to prevent crosstalk. We used trench refill and etch back [8], [13] approach for this, where isolation trenches are formed and completely filled with dielectric material (silicon dioxide in this case) and polysilicon.

The fabrication process given in Fig. 2 starts on SOI wafer with $50-\mu \mathrm{m}$-thick heavily doped device layer. First, $4-\mu \mathrm{m}$-wide isolation trenches are etched down to the buried oxide layer using the DRIE process and thermal oxidation follows to grow $1-\mu \mathrm{m}$-thick oxide on the sidewall of trenches for the electrical isolation in Fig. 2(a). In the next step, 2- $\mu$ m-thick undoped

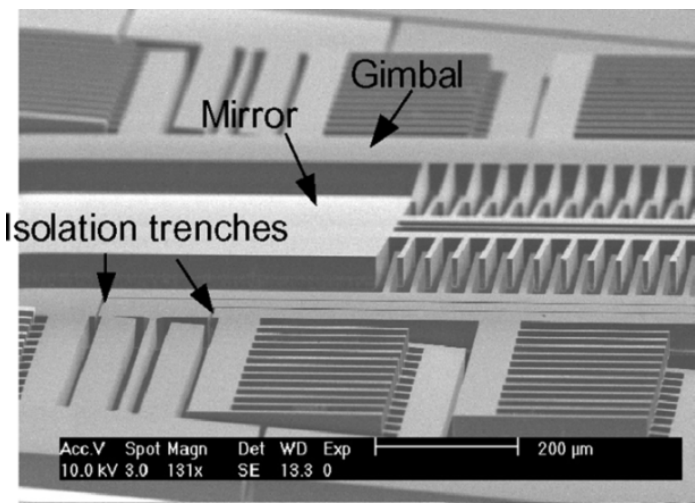

Fig. 3. SEM picture of close-up view of mirror, gimbal, and electrical isolation trenches.

polysilicon is deposited until the trenches are completely filled such that the structures divided by trenches are still mechanically connected with filled polysilicon after the polysilicon layer on the top surface is etched back in Fig. 2(b). In the following step in Fig. 2(c), the top- and bottom-combs, gimbal, torsion bars, and mirror plates on the front side and the openings on the backside are defined, etched, and released. A lid wafer is separately processed using a single step of DRIE with keys that self-align to key slots on the device wafer; and with pillars that displace both the gimbal and mirror to a desired tilt angle concurrently. In Fig. 2(d), the two-mated wafers are annealed in a furnace at $900^{\circ} \mathrm{C}$. When they are separated, both gimbals and mirrors are plastically deformed, as shown in Fig. 2(e), and the interdigitated comb fingers are vertically offset with self-alignment. The scanning electron microscope (SEM) photograph of the close-up view of one device is presented in Fig. 3 showing the tilted mirror, gimbal, and comb fingers as well as the isolation trenches.

\section{EXPERIMENTAL RESULTS}

The 2-D scanning mirror was tested using two independently controlled sinusoidal voltages from two power supplies for each axis of rotation. The images shown in Fig. 4 were generated from a single light beam reflected on 2-D scanning mirror and projected on a screen at various operational frequencies of gimbal and mirror. Depending on the ratio of the two driving frequencies and the phase difference of the two sinusoidal input voltages, different shapes of static and dynamic images are formed. For example, Fig. 4(a)-(c) are standing waves with fixed shapes and (d) is floating wave where the lines move constantly. The frequency response of the 2-D actuators are measured and presented in Fig. 5. Data were collected using $20-\mathrm{V}_{\mathrm{dc}}$ and $15-\mathrm{V}_{\mathrm{pp}}$ drive for mirror and $30-\mathrm{V}_{\mathrm{dc}}$ and $25-\mathrm{V}_{\mathrm{pp}}$ drive for gimbal. The resonant frequency is measured at $1.54 \mathrm{kHz}$ with a maximum scanning angle of $20^{\circ}$ for gimbal and $10.56 \mathrm{kHz}$ with a maximum scanning angle of $27^{\circ}$ for mirror and nonlinear responses are found in both directions. Among numbers of possible reasons of nonlinearity, electrostatic spring softening effect coming from the high voltages of operation and large motion is suspected to be the dominant source. To ensure the device reliability, both mirror and gimbal were resonated simultaneously at the aforementioned frequencies and voltages for 90 days, which corresponds to 82.0 billion cycles in the mirror axis and 12.1 billion cycles in the gimbal 


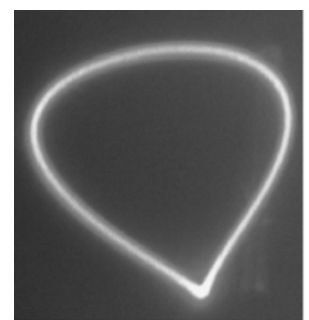

(a)

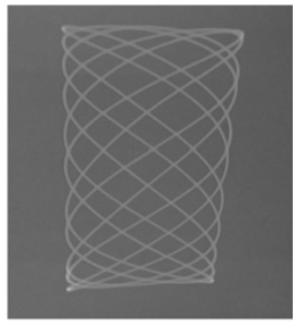

(c)

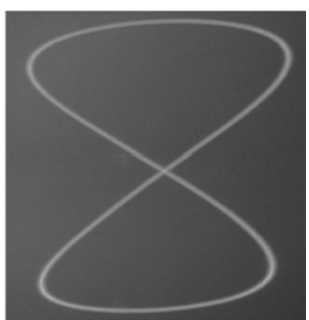

(b)

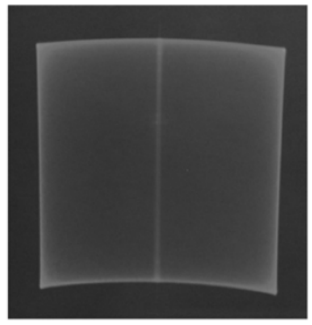

(d)
Fig. 4. Exemplary projected images generated by single light beam using 2-D scanning mirror actuators. (a)-(c) are standing waves with fixed shapes and (d) is floating wave and the lines moves constantly. (a) $f_{\text {mirror }} / f_{\text {gimbal }}=1$, (b) $f_{\text {mirror }} / f_{\text {gimbal }}=2$, (c) $f_{\text {mirror }} / f_{\text {gimbal }}=2.2$, (d) $f_{\text {mirror }} / f_{\text {gimbal }}=$ arbitrary number.
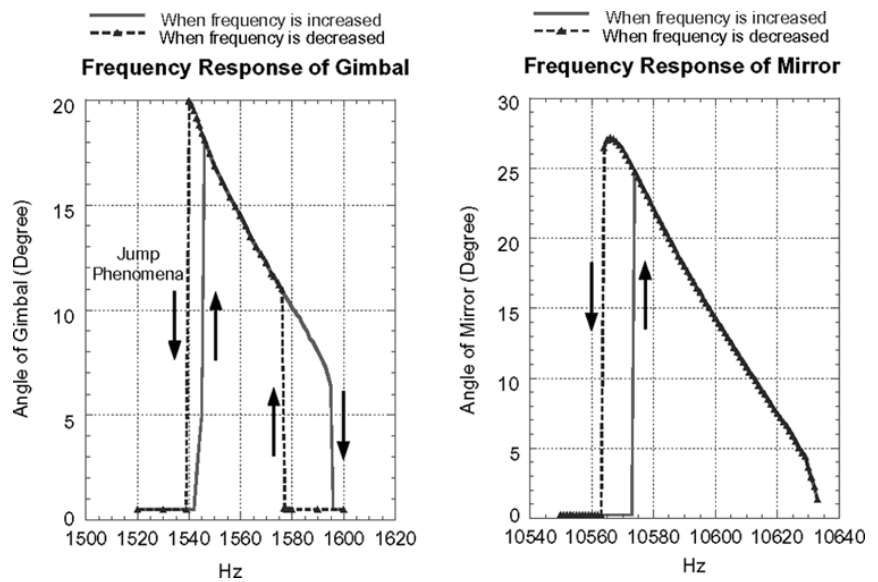

Fig. 5. Frequency response of gimbal and mirror of the 2-D scanning mirror actuator.

axis. The scanning angles were measured in each direction every seven days during the 90 days of full-time operation at resonance, and the angles decreased in both directions. Among the $0.98 \%$ decrease in mirror motion and $1.5 \%$ decrease in gimbal motion compared to the scanning angle at the beginning of the test, the majority of the angle changes were observed within the first week of operation and the decrease was monotonous at a very slow rate thereafter. The performance of 2-D scanning mirror actuator is summarized in Table I.

\section{CONCLUSION}

We have demonstrated microfabricated monolithic 2-D scanning mirrors using a three-mask process on SOI wafer with a single plastic deformation step. The mirror is electrostatically and independently actuated in two orthogonal directions by two sets of self-aligned angular vertical comb-drives.
TABLE I

SUMMARY OF 2-D SCANNING MiRROR ACTUATOR PERFORMANCES

\begin{tabular}{|c|c|c|}
\hline Actuation axis & Mirror & Gimbal \\
\hline Resonant frequency $(\mathrm{kHz})$ & $10.54 \mathrm{kHz}$ & $1.56 \mathrm{kHz}$ \\
\hline Quality factor & 503 & 73 \\
\hline Actuation voltage & $20 \mathrm{Vdc}+15 V_{p p}$ & $30 \mathrm{Vdc}+25 V_{p p}$ \\
\hline Optical scan angle & $27^{\circ}$ & $20^{\circ}$ \\
\hline $\begin{array}{l}\% \text { changes of scan angle after } 90 \\
\text { days of operation:( } 82.0 \text { billion } \\
\text { cycles for mirror, } 12.1 \text { billion } \\
\text { cycles for gimbal })\end{array}$ & $0.98 \%$ & $1.5 \%$ \\
\hline
\end{tabular}

Various forms of Lissajous patterns are demonstrated using a single light source and several different sets of actuation-frequency combinations of mirror and gimbal motions. The measured dynamic characteristics show resonant frequencies of 10.56 and $1.54 \mathrm{kHz}$ with optical scanning angles up to $27^{\circ}$ and $20^{\circ}$ with respect to mirror and gimbal axes, respectively. After 90 days of full-time operation, the scan angle deviations in each directional motion are negligible and we believe the 2-D actuator is reliable. To achieve the compatibility with the complementary metal-oxide-semiconductor process, the plastic deformation by the localized heating technique [4] is under development for 2-D actuators.

\section{REFERENCES}

[1] R. A. Conant, J. T. Nee, K. Lau, and R. S. Mueller, "A flat high-frequency scanning micromirror," in Solid-State Sensor and Actuator Workshop, Hilton Head, SC, 2000, pp. 6-9.

[2] U. Krishnamoorthy, D. Lee, and O. Solgaard, "Self-aligned vertical electrostatic combdrives for micromirror actuation," J. Microelectromech. Syst., vol. 12, pp. 458-464, 2003.

[3] J. Kim, H. Choo, L. Lin, and R. S. Muller, "Microfabricated torsional actuator by self-aligned plastic deformation," in 12th Int. Conf. SolidState Sensors, Actuators, and Microsystems, 2003, pp. 1015-1018.

[4] J. Kim and L. Lin, "Batch-fabricated scanning micromirrors using localized plastic deformation of silicon," in 17th Annu. IEEE Int. Micro Electro Mechanical Systems Conf., 2004, pp. 494-497.

[5] S. Schweizer, P. Cousseau, G. Lammel, S. Calmes, and P. H. Renaud, "Two-dimensional thermally actuated optical microprojector," Sens. Actuators A, Phys., vol. A85, no. 1-3, pp. 424-429, 2000.

[6] N. Asada, H. Matsuki, K. Minami, and M. Esashi, "Silicon micromachined two-dimensional galvano optical scanner," IEEE Trans. Magn., vol. 30, no. 6, pt. 1, pp. 4647-4649, Nov. 1994.

[7] Z. Hao, R. Clark, J. Hammer, M. Whitley, and B. Wingfield, "Modeling air-damping effect in a bulk micromachined 2-D tilt mirror," Sens. Actuators A, Phys., vol. A102, pp. 42-48, 2002.

[8] H. Schenk, P. Durr, D. Kunze, H. Lakner, and H. Kuck, "A resonantly excited 2D-micro-scanning-mirror with large deflection," Sens. Actuators A, Phys., vol. A89, pp. 104-111, 2001.

[9] W. Piyawattanametha, P. R. Patterson, D. Hah, H. Toshiyoshi, and M. C. Wu, "A 2-D scanner by surface and bulk micromachined vertical comb actuators," in 2003 IEEE/LEOS Int. Conf. Optical MEMS, 2003, pp. 93-94.

[10] S. Kwon, V. Milanovic, and L. P. Lee, "Vertical combdrive based 2-D gimbaled micromirrors with large static rotation by backside island isolation," IEEE J. Sel. Topics Quantum Electron., vol. 10, no. 3, pp. 498-504, May/Jun. 2004.

[11] V. Milanovic, M. Last, and K. S. J. Pister, "Torsional micromirrors with lateral actuators," in Transducers 2001, Munich, Germany, pp. 1290-1301.

[12] V. Milanovic, G. A. Matus, and D. T. McCormick, "Gimbal-less monolithic silicon actuators for tip-tilt-piston micromirror applications," IEEE J. Sel. Topics Quantum Electron., vol. 10, no. 3, pp. 462-471, May/Jun. 2004.

[13] D. Zhang, Z. Li, T. Li, and G. Wu, "A novel isolation technology in bulk micromachining using deep reactive ion etching and a polysilicon refill," J. Micromech. Microeng., vol. 11, pp. 13-19, 2001. 